\title{
Market Risk Measurement for Crude Oil: A Wavelet Based VaR Approach
}

\author{
Kin Keung LAI, Kaijian HE, Chi XIE, and Shou CHEN
}

\begin{abstract}
With the development of technology and financial engineering tools, oil markets are more competitive and volatile than ever before. This places the accurate and reliable measurement of market risks in the crucial position for both investment decision and hedging strategy designs. This paper tackles the measurement of risks from a Value at Risk (VaR) perspective. Since traditional ARMA-GARCH approach doesn't suffice, this paper proposes ex-ante based approach for hybrid algorithm design and further applies this methodology with a wavelet approach to VaR estimates. Empirical studies of the proposed Wavelet Decomposed Value at Risk (WDVaR) have been conducted on two major oil markets (I.e. WTI \& Brent). Experiment results suggest that the performance of WDVaR improves upon ARMA-GARCH model at higher confidence levels. Meanwhile, WDVaR offer considerable flexibility during modeling process. WDVaR can be tailored to specific market characteristics and its performance can be further improved with more careful parameter tuning.
\end{abstract}

\section{INTRODUCTION}

$\mathrm{T}$ HE crude oil is one of the most important industry inputs and remains the major sources of world's energy consumption. The price paths of crude oil and its volatilities affect different market movements (E.g. various commodities markets, etc) and the economic status as a whole[1], [2]. Oil markets have long been the most volatile ones since shocks and the associated risks of losses could prevail in the market due to low inventory level hindered by extremely high storage costs. As the role of market forces increase continuously with the shifts of market from more managed market agreement to the more flexible market based environment, market is getting more volatile and vulnerable to unexpected extreme events[2]. Thus proper measurement and management of market risks are increasingly valued by investors to protect themselves against adverse market movements.

This paper investigates the risk measurement issue in oil markets. The measurement of risks in oil markets are complicated processes since oil prices receive joint influences from numerous risk factors. To name just a few, these may include economic aspects, weather changes, political aspects,

K. K. Lai is with the College of Business Administration, Hunan University, Changsha, Hunan, China. He is also with the Department of Management Sciences, City University of Hong Kong, Tat Chee Avenue, Kowloon, Hong Kong (phone: +852 2788 8563; fax: +852 27888560 ; email: mskklai@cityu.edu.hk).

KJ. HE is with the College of Business Administration, Hunan University, Changsha, Hunan, China (email: paulhekj@hnu.cn).

C. Xie is with the College of Business Administration, Hunan University, Changsha, Hunan, China (email: xiechi@hnu.cn)

$\mathrm{S}$. Chen is with the College of Business Administration, Hunan University, Changsha, Hunan, China (email: chenshou@hnu.cn)

U.S. Government work not protected by U.S. copyright military, natural disasters, market sentiments and speculations, etc[3], [4], [5]. If these factors and their interrelationships can be identified and quantified, large scale econometric models can be built to understand and track risk exposure levels. However, in practice this approach is generally very costly and infeasible except for rare circumstances. Another approach would resolve to the reduced form model such as time series analysis for help. With reduced form model, information is extracted from the past data and used to guide the future forecasting. This approach is less costly and more suitable when specific information relating the underlying risk factors and their interactions are not available[5]. The methodology used in this paper follows the reduced form approach.

Value at Risk (VaR), as the latest development in the risk management field, is adopted in this paper to quantify and measure market risks. VaR is a single, summarizing statistic number that measures the magnitude of downside risk under normal market conditions over certain investment horizon at given confidence levels[6]. The reliability and accuracy of VaR estimates are of particular interests to investors since they would affect their capital adequacy and profit level. On the other hand, oil markets, among various commodity markets, attract significant research efforts in recent years due to the following reasons: Firstly data have been made publicly available at sufficiently high frequency and long time period. This makes possible the development and testing of $\mathrm{VaR}$ estimates under statistical framework[7], [8]. Secondly, price series in oil markets exhibit much higher level of volatility than other commodities and stock markets. The considerable risks and unique characteristics of the market demand the development of new risk management tools[7], [8]. Thirdly despite the proliferation of volatility analysis and forecasting of oil prices, the open literature is surprisingly scarce in the development of appropriate risk measurement for the market. There have been few attempts to uncover the stylized features of oil markets, not to mention the estimation issue that lies at the heart of $\mathrm{VaR}$ implementation [3], [4], [9], [10], [11].

To estimate $\mathrm{VaR}$ at reliable and accurate level, various methods have been tried over years. They can be broadly categorized into parametric and non-parametric approaches. Non-parametric approaches give investors little insights and controls over underlying risk factors evolutions. Parametric approaches are ex-post in nature. They fit model into data with the hope that the data are mostly dominated by the expected data features and the model should be able to pick them up. Since this rarely holds in practice, their 
performances usually deteriorates rapidly once they are outside their original problem domain[12], [13], [14], [15], [16].

Therefore, this paper introduces ex ante approach for hybrid algorithm design to offer deeper insights and more flexibility during modeling process. As an application, the wavelet analysis is introduced into hybrid based approach for VaR estimates. Empirical studies based on the proposed WDVaR algorithm \& more traditional ARMA-GARCH approach is conducted in both WTI and Brent oil markets. Experiment results are backtested and compared using Kupiec backtesting procedures to evaluate their accuracy and reliability[17]. Incorporating the flexibility of ex ante hybrid algorithm and details analysis in time scale domain offered by wavelet analysis, WDVaR is found to improve the reliability of VaR estimates and offer greater flexibility than traditional ARMA-GARCH approach.

The organization of the paper develops as follows: the second section reviews the relevant literature in VaR and wavelet theory. This section also identifies and reviews the relevant wavelet applications in economics and finance field. The third section proposes the ex-ante approach for hybrid algorithm design. WDVaR is proposed as a specific application of wavelet analysis to $\mathrm{VaR}$ estimates. The empirical studies in two major oil markets are conducted. The performance of the proposed model and the more traditional ARMA-GARCH based model are evaluated and compared. And the fifth section concludes.

\section{LITERATURE REVIEW}

\section{A. Value at Risk (VaR)}

As defined mathematically in (1), VaR provides the statistical measurement of the maximum losses (risks) $r_{V a R}$ over a certain investment horizon $\mathrm{t}$ under normal market conditions and the given the confidence level [6].

$$
p\left\{\Pi(0)-\Pi(t) \leq-r_{V a R}\right\}=1-c l
$$

Where $\Pi(\bullet)$ is the value of the portfolio at time $t$. $t$ is the holding period and $\mathrm{cl}$ is the confidence level.

Based on the way information is extracted from historical data, the estimation of VaR has been tackled from three different approaches over years. Namely: parametric, non-parametric and semi parametric.

Parametric approaches try to fit data into presupposed distribution and derive tractable analytic forms. These include popular econometric tools such as ARMA and GARCH models, etc. They provide deeper insights into risk evolutions and are generally computationally more convenient during implementation. However, their performances are highly variable and sensitive to data samples because it usually imposes strict assumptions during modeling processes[18]. Non-parametric approaches make no assumptions as to the underlying risk distributions and analytic forms. These include techniques such as historical simulation, bootstrap methods, kernel methods, Monte Carlo simulation methods, principal component analysis (PCA), and factor analysis, etc. They are flexible in picking up unknown data features. However, non-parametric approaches itself offer little insights and control over the underlying risk evolution, and thus, are inferior to parametric approaches when sufficient statistical justification is needed for model's implementation[18], [19], [20], [21], [22]. Recently semi-parametric approaches emerge as the attempts to balance between two extremes. These include emerging tools such as extreme value theory (EVT), Fourier transformation, wavelet transformation, fuzzy logic etc. Current results from empirical studies are quite mixed[20], [23], [24].

In this paper, traditional hybrid algorithm based on ARMA-GARCH model serves as the benchmark model[25], [26]. The usual ARMA(r, m)-GARCH(p,q) takes the form as in (2):

$$
\begin{aligned}
& r_{t}=\alpha_{0}+\sum_{i=1}^{r} \alpha_{t-i}+\varepsilon_{t}+\sum_{j=1}^{m} b_{j} \varepsilon_{t-j} \quad \varepsilon_{t} \in N\left(\mu, \sigma_{t}\right) \\
& \sigma_{t}=\omega+\sum_{i=1}^{p} \alpha_{i} \varepsilon_{t-i}^{2}+\sum_{j=1}^{q} \beta_{j} \sigma_{t-j}^{2}
\end{aligned}
$$

Among various parametric models available, hybrid ARMA model with GARCH error correction is widely accepted in both academic and industries since it's easy to understand and implement in practice. But its performance is constrained by its sequential linear filtering process as described before.

The appropriate lag order for the model is determined by information criteria with the visual aid of autocorrelation and partial autocorrelation functions. There are numerous information criteria available such as Akaike's information criterion (AIC), Schwarz's Bayesian information criterion (BIC), and the Hannan-Quinn information criterion (HQIC), etc[27], [28], [29]. This paper adopts commonly accepted AIC and BIC to test whether allowing dynamic order selection would bring significant performance improvement. The AIC and BIC are specified as in (3):

\section{Minimize AIC (or BIC)}

$$
\left\{\begin{array}{l}
A I C(\ell)=\frac{-2}{T} \ln (\gamma)+\frac{2 \ell}{T} \\
\text { or } B I C(\ell)=\frac{-2}{T} \ln (\gamma)+\frac{\ell}{T} \ln T
\end{array}\right.
$$

Where $\ell$ is the maximum likelihood estimates. $T$ is the number of parameters.

Backtesting procedures are standard framework to evaluate and compare the $\mathrm{VaR}$ estimation performance of various approaches. It essentially verifies whether the projected maximum losses are in line with actual losses. There are mainly two approaches to conduct backtesting procedures: hypothesis or forecasting based approaches[6]. Forecasting based approaches utilize the rewarding and losses function to 
evaluate more accurately how the model has performed at each step. Although it's very convenient when data size is small, it is not gaining its momentum since statistical confidence level is not given. Thus hypothesis based approaches are more desirable in practice. The null hypothesis for hypothesis based approaches is: the failure rate equals the theoretical losses, which implies that the estimates accuracy from the tested model is statistically significant and the model is acceptable under the given confidence level[30]. The testing steps are treated as a series of Bernoulli trials. And the failure rates should converge to the binomial distribution if the sample size is large enough and the model is acceptable under given confidence levels. The likelihood ratio test statistics takes the form as in (4).

$$
L R_{u c}=-2 \log \left[(1-\rho)^{T-N} \rho^{N}\right]+2 \log \left\{\left[1-\left(\frac{N}{T}\right)\right]^{T-N}\left(\frac{N}{T}\right)^{N}\right\}
$$

Where $L R_{u c}$ denotes the test statistics that has an asymptotic $\chi^{2}(1)$ distribution. $\mathrm{T}$ is the total number of observations used in test set. $\rho$ is the probability of an VaR exceedance occurrence. The critical value for test statistics is 3.8415 at $95 \%$ confidence level. The model is only acceptable at $95 \%$ confidence level when test statistics is lower than the critical value.

\section{B. Wavelet Analysis}

Wavelets are families of functions that satisfy admissibility condition and defined as in (5)

$$
C_{\psi}=\int_{0}^{\infty} \frac{|\varphi(f)|}{f} d f<\infty
$$

Where $\varphi(f)$ is the Fourier transform of wavelet $\Psi(t)$ in the frequency domain.

Compared to sinusoids used in Fourier analysis to spot frequency domain features, wavelets have vanishing moments and exhibit the behavior of 'small wave' that has its energy concentrated in a short interval of time[31], [32]. There are different wavelets families available. These may include Haar wavelets, Daubechies wavelet, Minimum Bandwidth Discrete-Time Wavelets (MBDT) etc[31], [32], [33] Among them, Haar wavelets is the only symmetric compactly supported orthonormal wavelet [34]. Daubechies wavelet can be viewed as a generalized version of Haar wavelet[23]. Different wavelet families could accentuate different data characteristics in time scale domain and serve as a potential pattern recognition tool.

Therefore, wavelet analysis uses different wavelets to decompose and capture specific data characteristics in time scale domain. In wavelet analysis, scaling filters are applied to capture low frequency component of the signal, which corresponds to trends in data. Meanwhile, wavelet filter is applied at different scales to capture higher frequency components of the signal, which correspond to the short lived and transient behavior in data[35], [36]. The separation of data components that are unique in time scale domain could potential reveal the different risk factors in the market place.
These decomposed parts may correspond to investors with particular investment horizon and strategies.

Since wavelet transform is orthonormal, signals can be decomposed and reconstructed perfectly using wavelet analysis. Wavelet analysis can be used to perform multi resolution analysis on signals in time scale domain. The decomposition of the original return series $x(t)$ is conducted as in (6) to reveal interesting characteristics.

$$
W(u, s)=\int_{-\infty}^{\infty} x(t) \psi_{u, s}(t) d t
$$

Where $\psi_{u, s}(t)=\frac{1}{\sqrt{s}} \Psi\left(\frac{t-u}{s}\right), \mathrm{u}$ is the wavelet parameter translating the original wavelet function, $\mathrm{s}$ is the scale parameter dilating the original wavelet function.

The reconstruction (or synthesis) of the original return series from decomposed wavelet coefficients is performed as in (7)

$$
x(t)=\frac{1}{C_{\Psi}} \int_{0}^{\infty} \int_{-\infty}^{\infty} W(u, s) \Psi_{u, s}(t) d u \frac{d s}{s^{2}}
$$

\section{Wavelet Applications in Economics and Finance}

The application of wavelet analysis in economic and financial data analysis is only recent phenomenon. Since wavelet analysis projects data signals into time scale domain for analysis, it can be treated as promising multi scale analysis, noise reduction and multi scale modeling tool.

Firstly, if wavelet analysis is introduced as multi scale analysis framework, it can be utilized to reveal some interesting economic relationship and financial data behavior that are not visible in the traditional frequency domain [37], [38], [39], [40]. Secondly, the decomposition capability also evolves into efficient noise reduction tool. The so called wavelet shrinkage techniques can be used to clean up the distorted economic and financial data for further processing. This would result in higher level of model fit and forecasting accuracy, as confirmed by empirical studies conducted in both electricity and foreign exchange markets [41], [42]. Thirdly, the decomposition capability could also be directly integrated into the modeling process. The original data is decomposed before further modeling attempts are applied. The multi scale wavelet based approaches achieve performance improvement through finer process modeling at each individual scales. [43], [44], [45]. This is confirmed by results from empirical studies in both electricity and energy markets. However, in its current stage of development, this approach is carried out on a largely arbitrary basis and lacks theoretical justification[46], [47], [48], [49].

Compared to the scattered, but gradual progress researchers have made in the application of wavelet theory, there is surprisingly less relevant literature identified in the risk management field. To the best of our knowledge, only three attempts have been identified to apply wavelet analysis in risk measurement[50], [51], [52]. These works are mainly concerned with the decomposition of risks over different time horizons to investigate risk distributions. The proportion of 
risks over the time horizons that correspond to investors' investment strategies are supposed to be fixed. And the future estimated risk exposure level is adjusted by this fixed ratio to reflect the risks investors are interested in. However the key issue of how to estimate risk measurement at higher accuracy and reliability has been left intact for their approaches of applying wavelet theories in risk management.

\section{WAVELET DECOMPOSED VALUE AT RISK (WDVAR)}

\section{A. WDVaR background}

According to the heterogeneous market hypothesis, risks reflect the joint influence from various risk factors evolving in different time horizons. Traditionally various models have been attempted to describe the complex risk evolution process. They aim to capture particular data characteristics. This results in the quick deterioration of model's performance once they are outside the problem domain being investigated. Although a handful of statistical tests have been utilized to help in identifying the existence of particular data characteristics, these tests usually lack sufficient discriminatory power for noisy data and may not cover all the data features under investigation.

Thus, hybrid and ensemble approaches have received considerable attentions recently. Hybrid algorithm linearly filters the data through different model mechanisms ranked by their significance. It's simple to implement and easy to understand. But it fails to take into account the time varying nature of parameters. ARMA-GARCH is the dominant hybrid algorithm and serves as the benchmark model in this paper. Meanwhile, ensemble algorithm takes a different approach by firstly trying to fit different models to the data. And then the weight of their significances is nonlinearly determined using artificial intelligent models such as neural network, etc. It has achieved certain level of performance improvement, but its performance is still theoretically constrained by how accurately each tried model is capable of capturing specific data features from noisy environment[53], [54], [55].

More generally, previous approaches usually relied on the assumptions that individual model could fully extract the targeted data feature. This is less of an issue when these algorithms are tried in the environment where data features of interest are less distorted. But recent empirical studies have identified the rapid deterioration of their performance once they are outside their original problem domain. When data features of interest less dominant, the forecasting accuracy of each individual forecaster is challenged. The ex-post nature of previous approaches leave them prone to noises and errors in data, which made them unreliable in practice.

Thus this paper takes a different ex-ante approach and introduces signal processing techniques as a promising direction for hybrid and ensemble algorithm design. This paper contributes to the existing literature by shifting the focus from ex-post filtering struggles to ex-ante decomposition and classification. The proposed ex-ante approach is semi parametric in nature. As is commonly found in various engineering disciplines such as image processing, the proposed ex-ante approach makes no assumptions about data distributions and patterns in the first place and use signal processing techniques to investigate the underlying risk compositions.

\section{B. WDVaR Framework}

When the data distribution can be characterized using the first and second moment, the $\mathrm{VaR}$ is estimated following (8)

$$
\operatorname{VaR}=-F(a) \hat{\sigma}_{t+1 \mid t}-\hat{\mu}
$$

Where $F$ (a) refers to the corresponding quantile (95th, 97.5th or 99th) of the assumed distribution (The elliptical distribution is assumed in this paper due to large sample size involved). $\hat{\sigma}_{t+1 \mid t}$ refers to the forecast of conditional standard deviation at time $\mathrm{t}+1$ given information at time $\mathrm{t} . \widehat{\mu}$ refers to the forecast of sample mean. Outside the elliptical realm, the first and second may not be enough to characterize the data distribution. Thus more complex parametric VaR form is needed and is beyond the scope of this paper.

Based on the multi resolution analysis principle, the original data returns can be decomposed into different time scales using wavelet analysis as in (9)

$$
f(t)=f_{A^{j}}(t)+\sum_{j=1}^{J} f_{D^{j}}(t)
$$

Where $\mathrm{f}(\mathrm{t})$ is the original signal. $f_{A^{J}}(t)$ is the decomposed time series data by applying scaling function at scale j. $f_{D^{j}}(t)$ is the decomposed time series data by applying wavelet function at scale $\mathrm{j}$.

Since VaR estimates is additive in elliptical realm, given that wavelet analysis decorrelates the original data, the VaR estimated to cover portfolio losses is also expected to cover losses at each individual scales as in (10):

$$
\operatorname{VaR}=\operatorname{VaR} R_{A^{j}}+\sum_{j=1}^{J} \operatorname{VaR} R_{D^{j}}
$$

Expanding (10) into (11)

$$
V a R=-\left(F(a) \hat{\sigma}_{t+1 \mid t, A^{j}}+\hat{\mu}_{A^{j}}\right)-\sum_{j=1}^{J}\left(F(a) \hat{\sigma}_{t+1 \mid t, D^{j}}+\hat{\mu}_{D^{j}}\right)
$$

Therefore, the estimation of WDVaR boils down to the estimation of conditional mean and conditional volatility [17].

The conditional mean is modeled as ARMA ( $\mathrm{r}, \mathrm{m})$ ( $\mathrm{r}$ and $\mathrm{m}$ is the lag order) process. This is given in (12) 


$$
\widehat{\mu}=\frac{1}{n} \sum f(t)=\frac{1}{n} \sum f_{A^{j}}(t)+\sum_{j=1}^{J} \frac{1}{n} \sum f_{D^{j}}(t)=\widehat{\mu_{A^{j}}}+\sum_{j=1}^{J} \widehat{\mu_{D^{j}}}
$$

The conditional volatility is modeled as a mixture of GARCH $(1,1)$ processes at each scale. This is also given in (13).

$$
\hat{\sigma}=\hat{\sigma}_{t+1 l t, A^{j}}+\sum_{j=1}^{J} \hat{\sigma}_{t+1 l t, D^{j}}
$$

\section{PROCEDURE FOR PAPER SUBMISSION}

\section{A. Data Set and Data Pre-processing}

West Texas Intermediate (WTI) and Brent (or Brent-Forties-Oseberg) crude oil spot prices are used in this study. These markets are considered the world marker crude oil markets. Most other crude oil prices are related to them. The data set includes 4741 observations. The covered period is: from 20th May, 1987 to 30th December, 2005 (for Brent crude oil) and from 27th March, 1987 to 30th December, 2005 (for WTI crude oil) respectively. $60 \%$ of the data set (i.e. 2845 ) serves as the training set, while the remaining $40 \%$ of the data set (i.e. 1896 observations) is used as the test set. One step ahead out of sample forecast is conducted to evaluate the accuracy and reliability of various models under investigation. During implementation, the training set is continuously expanded to include the newly available observation at each iteration, so that the arrival of new information is taken into consideration. A portfolio of one asset position worth $\$ 1$ is assumed for each market. The original observations are log differenced (i.e. $\log \left(x_{t}\right)-\log \left(x_{t-1}\right)$ ) for further processing and modeling attempts.
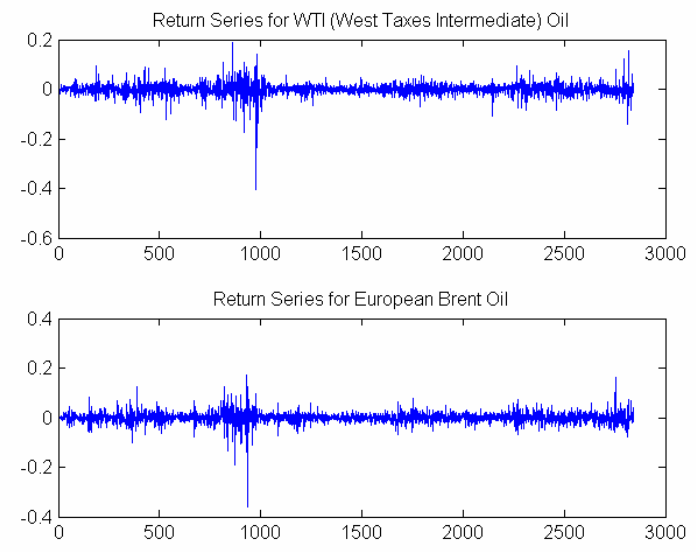

Fig. 1. Return Series for Crude Oil Markets

\section{B. Descriptive Statistics and Hypothesis Testing}

The oil markets are characterized by high volatility and the leptokurtic phenomenon (i.e. fat tail and high kurtosis, which signals high probability of extreme events occurrences), which makes adequate risk management and control necessary. This is confirmed by several stylized facts concluded from table I:

Firstly, both markets are highly volatile. There are significant price fluctuations in the markets as suggested by positive standard deviations. The substantial difference between the minimum and maximum level also indicates considerable losses in both markets, as investors could face TABLE I

DESCRIPTIVE STATISTICS \& STATISTICAL TESTS IN WTI \& BRENT CRUDE

\begin{tabular}{|c|c|c|}
\hline Oil Markets & $\begin{array}{l}\text { WTI(West Texas } \\
\text { Intermediate) }\end{array}$ & Brent \\
\hline Mean & -0.0001 & -0.0001 \\
\hline Maximum & 0.1887 & 0.1733 \\
\hline Minimum & -0.4063 & -0.3612 \\
\hline Medium & 0 & 0 \\
\hline $\begin{array}{l}\text { Standard } \\
\text { Deviation }\end{array}$ & 0.0240 & 0.0226 \\
\hline Skewness & -1.7343 & -1.3071 \\
\hline Kurtosis & 37.9541 & 32.9762 \\
\hline \multicolumn{3}{|l|}{ Jarque-bera } \\
\hline $\begin{array}{l}\text { Test (p } \\
\text { value) }\end{array}$ & 0 & 0 \\
\hline $\begin{array}{l}\text { BDS Test } \\
\text { (p value) }\end{array}$ & 0 & 0 \\
\hline
\end{tabular}
OIL MARKETS

large gains as well as huge losses if risks are not properly measured and managed.

Secondly, there is generally higher probability of losses in these markets as indicated by the negative skewnesses.

Thirdly, risks also appear in the form that extreme events prevail in these markets as suggested by high excess kurtosis. It appears that excess kurtosis found in oil markets are much higher than those normally found in the stock markets. This implies a more risky environment, where the short lived and transient behavior could hit investors with surprises and causes huge losses if proper risk controls techniques are not in place.

The nonlinear and volatile nature of the oil markets are further confirmed by formal statistical tests conducted. The rejection of Jarque-bera test of normality suggests that the returns deviate from normal distribution significantly and exhibit leptokurtic behaviors. The rejection of BDS (Brock-Dechert-Scheinkman) test of independence indicates the existence of non-linearity within the data $[56]^{1}$.

\section{Forecast Performance Results}

\section{1) ARMA-GARCH VaR}

Although dynamic order selection based on information criteria (E.g. AIC or BIC etc) is found to improve forecasting accuracy slightly as suggested by results in table II and III, the associated computational complexity increases exponentially. Thus in this paper $\operatorname{ARMA}(1,1)$ is chosen to model conditional mean and $\operatorname{GARCH}(1,1)$ is chosen to model conditional heteroskedasticity. The estimation for $\operatorname{ARMA}(1,1)-G A R C H(1,1)$ hybrid model is based on rolling-window method (the window length is set to 2843 to cover the initial information set available).

As suggested by experiment results, ARMA(1,1)-GARCH(1,1) performs rather well. It only fails

\footnotetext{
1 The implementation of BDS (Brock, Dechert\& Scheinkman (1986)) test for independence based on the correlation dimension follows L.Kanzler's approach. http://www2.gol.com/users/kanzler/index.htm
} 
TABLE II

EXPERIMENT RESULTS FOR ARMA-GARCH VAR IN OIL MARKETS

\begin{tabular}{lcccccc}
\hline \hline \multirow{2}{*}{$\begin{array}{l}\text { Oil } \\
\text { Markets }\end{array}$} & \multicolumn{3}{c}{$\begin{array}{c}\text { WTI(West Texas } \\
\text { Intermediate) }\end{array}$} \\
\cline { 2 - 7 } & $95.0 \%$ & $97.5 \%$ & $99 \%$ & $95.0 \%$ & $97.5 \%$ & $99 \%$ \\
\hline $\begin{array}{l}\text { ARMA-GA } \\
\text { RCH VaR }\end{array}$ & 89 & 59 & 39 & 97 & 54 & 27 \\
$\begin{array}{l}\text { Exceedance } \\
\text { MSE (Mean }\end{array}$ & & & & & & \\
$\begin{array}{l}\text { Square } \\
\text { Errors) }\end{array}$ & 0.0027 & 0.0035 & 0.0047 & 0.0024 & 0.0031 & 0.0041 \\
$\begin{array}{l}\text { Kupiec Test } \\
\text { Statistics }\end{array}$ & 0.3810 & 2.7049 & 16.3907 & 0.0534 & 0.9027 & 3.0438 \\
$\begin{array}{l}\text { P Value } \\
\text { ARMA-GA } \\
\text { RCH Model }\end{array}$ & 0.5371 & 0.1000 & 0.0001 & 0.8173 & 0.3421 & 0.0810 \\
Acceptance & $\sqrt{ }$ & $\sqrt{ }$ & $\times$ & $\sqrt{ }$ & $\sqrt{ }$ & $\sqrt{ }$
\end{tabular}

TABLE III

EXPERIMENT RESULTS FOR ARMA(1,1)-GARCH(1,1) VAR IN OIL MARKETS

\begin{tabular}{lcccccc}
\hline \hline \multirow{2}{*}{$\begin{array}{l}\text { Oil } \\
\text { Markets }\end{array}$} & \multicolumn{3}{c}{ WTI(West Texas } & \multicolumn{3}{c}{ Brent } \\
\cline { 2 - 7 } & $95.0 \%$ & $97.5 \%$ & $99 \%$ & $95.0 \%$ & $97.5 \%$ & $99 \%$ \\
\hline $\begin{array}{l}\text { ARMA(1,1 } \\
\text { )-GARCH( }\end{array}$ & 90 & 59 & 38 & 97 & 54 & 27 \\
$\begin{array}{l}1,1) \text { VaR } \\
\text { Exceedance }\end{array}$ & & & & & & \\
$\begin{array}{l}\text { MSE } \\
\text { (Mean }\end{array}$ & 0.0027 & 0.0035 & 0.0047 & 0.0024 & 0.0031 & 0.0041 \\
$\begin{array}{l}\text { Square } \\
\text { Errors) }\end{array}$ & & & & & & \\
$\begin{array}{l}\text { Kupiec Test } \\
\text { Statistics }\end{array}$ & 0.2600 & 2.7049 & 14.9531 & 0.0534 & 0.9027 & 3.0438 \\
$\begin{array}{l}\text { P Value } \\
\text { ARMA(1,1 } \\
\text {-GARCH( }\end{array}$ & 0.6101 & 0.1000 & 0.0001 & 0.8173 & 0.3421 & 0.0810 \\
$1,1)$ Model & $\sqrt{ }$ & $\sqrt{ }$ & $\times$ & $\sqrt{ }$ & $\sqrt{ }$ & $\sqrt{ }$ \\
Acceptance & & & & & &
\end{tabular}

at $99 \%$ confidence level in WTI oil market and is accepted under all other circumstances. The performance of $\operatorname{ARMA}(1,1)-G A R C H(1,1)$ gradually deteriorates under higher confidence levels for both markets. ARMA(1,1)-GARCH $(1,1)$ provides much better coverage of risks under lower confidence level. This implies that ARMA(1,1)-GARCH $(1,1)$ model may underestimate risk measurement and serve as a generally aggressive risk measures. Since dynamic lag order selection can't improve performance much further, current linear hybrid approach for VaR estimates leaves little room for further performance improvement. Thus algorithms with greater flexibility are needed to describe the complicated underlying data generating processes.

2) $\operatorname{WDVaR}($ Haar, 2)

Two new parameters are introduced during WDVaR estimates: wavelet family chosen and the decomposition level. Therefore the notion $\operatorname{WDVaR}(\mathrm{x}, \mathrm{i})$ refers to the WDVaR algorithm that uses the chosen wavelet family $\mathrm{x}$ with the decomposition level $\mathrm{i}$. In this section, Haar wavelet families are chosen and the decomposition level is set to 2 . ARMA(1,1)-GARCH $(1,1)$ model is chosen to model the conditional mean and conditional volatility at each scale. The estimation for ARMA $(1,1)-\operatorname{GARCH}(1,1)$ hybrid model is based on rolling-window method (the window length is set to 2843 to cover the initial information set available)
TABLE IV

EXPERIMENT RESULTS FOR WDVAR(HAAR,2) IN OIL MARKETS

\begin{tabular}{lcccccc}
\hline \hline \multirow{2}{*}{$\begin{array}{l}\text { Oil } \\
\text { Markets }\end{array}$} & \multicolumn{3}{c}{$\begin{array}{c}\text { WTI(West Texas } \\
\text { Intermediate) }\end{array}$} \\
\cline { 2 - 7 } & $95.0 \%$ & $97.5 \%$ & $99 \%$ & $95.0 \%$ & $97.5 \%$ & $99 \%$ \\
\hline $\begin{array}{l}\text { WDVaR(H } \\
\text { aar, 2) }\end{array}$ & 68 & 46 & 25 & 76 & 43 & 24 \\
$\begin{array}{l}\text { Exceedance } \\
\text { MSE (Mean }\end{array}$ & & & & & & \\
$\begin{array}{l}\text { Square } \\
\text { Errors) }\end{array}$ & 0.0036 & 0.0047 & 0.0063 & 0.0034 & 0.0044 & 0.0057 \\
$\begin{array}{l}\text { Kupiec Test } \\
\text { Statistics }\end{array}$ & 8,8092 & 0.0428 & 1.7667 & 4.1981 & 0.4322 & 1.2482 \\
$\begin{array}{l}\text { P Value } \\
\text { WDVaR(H }\end{array}$ & 0.0030 & 0.8361 & 0.1838 & 0.0405 & 0.5109 & 0.2639 \\
$\begin{array}{l}\text { aar, 2) } \\
\text { Model }\end{array}$ & $\times$ & $\sqrt{ }$ & $\sqrt{ }$ & $\times$ & $\sqrt{ }$ & $\sqrt{ }$ \\
Acceptance & & & & & &
\end{tabular}

As confirmed by experiment results in table IV, wavelet based approach improves the reliability of VaR estimates at high confidence levels. WDVaR(Haar, 2) is accepted at 99\% confidence level in WTI markets. The reliability of VaR estimates are also improved for both $99 \%$ and $97.5 \%$ confidence level as indicated by higher $\mathrm{p}$ value in both markets. Thus WDVaR(Haar, 2) is less aggressive than ARMA(1,1)-GARCH(1,1) approach and provides better coverage of market risks at higher confidence levels.

This finding suggests that the time scale characteristic of risks in oil markets needs to be taken into consideration during modeling attempts to avoid underestimates. Single ARMA-GARCH model captures majority of risks, but may ignore substantial risks at finer details. Another implication would be that the nonlinear dependence or even chaos within data reflects the joint influences from various underlying processes. There may be multiple ARMA-GARCH processes with different lags evolving at individual scales. Proper mixing of ARMA-GARCH model could improve the model's performance significantly. Thus the selection of appropriate decomposition techniques is crucial to the understanding and further modeling of the complex market behavior.

Meanwhile, the performance of WDVaR at lower confidence level does deteriorate compared to the performance of ARMA-GARCH model. i.e. The $\mathrm{p}$ values are lower and WDVaR(Haar, 2) is rejected at $95 \%$ confidence levels in both markets. WDVaR(Haar, 2) seems to provide more conservative coverage of market risks. This is also confirmed by higher MSE (Mean Squared Errors) for WDVaR(Haar, 2). This finding indicates the subtleties in underlying risk compositions and the inadequacy of current models. However, with wavelet analysis introduced into risk management field, performance can be further improved by fine tuning parameters toward market characteristics. E.g. experiments based on WDVaR(Haar, 3) improves the reliability of VaR estimates at $99 \%$ confidence levels for both markets. The $p$ value increased to 0.5109 for WTI oil markets and 0.645 for Brent oil markets.

\section{CONCLUSIONS}

This paper discusses the limitations of current hybrid and 
ensemble approaches for time series forecasting. Then based on heterogeneous hypothesis for market microstructure, this paper applies wavelet analysis to value at risk estimates in oil markets and improve the risk measurement reliability at higher confidence levels. The contribution of this paper is twofold: firstly this paper proposes the ex-ante approach for hybrid algorithm design in contrast to the common ex-post filtering approach. The proposed methodology is applied in risk measurement field and the wavelet decomposed Value at Risk algorithm (WDVaR) is proposed. WDVaR has demonstrated its capability to improve the reliability of VaR estimates at higher confidence levels in both WTI and Brent oil markets. In addition, WDVaR offers more insights and considerable flexibility during underlying risk factors decomposition and modeling processes. It could be tailored to specific market environment and accentuate market characteristics of interests (E.g. specific investment strategies of interests, different investment time horizon in the market, etc). Secondly, this research paves the way for more comprehensive data analysis at finer details. By tuning parameters tailored for specific market characteristics, WDVaR offers additional flexibility and more insights into the underlying risk composition and evolutions.

\section{REFERENCES}

[1] C.W. Yang, M.J. Hwang, and B.N.Huang, "An Analysis of Factors Affecting Price Volatility of the US Oil Market," Energy Economics, vol. 24, pp. 107-119, 2002.

[2] A. Plourde and G,C Watkins, "Crude Oil Prices Between 1985 and 1994: How Volatile in Relation to Other Commodities?," Resources and Energy Economics, vol. 20, pp. 245-262, 1998.

[3] J. Alvarez-Ramirez, A. Soriano, M. Cisneros, and R. Suarez, "Symmetry/anti-symmetry phase transitions in crude oil markets," Physica A, vol. 322, pp. 583-596, 2003.

[4] S. Namit, "Forecasting Oil Price Volatility", master's thesis, Dept. Economics, Virginia Polytechnic Institute and State University, , Blacksburg, V.A.: 1998.

[5] S.Y. Wong, L.A. Yu, and K.K. Lai, "A Rough-Set-Refined Text Mining Approach for Crude Oil Market Tendency Forecasting," Int'l J. Knowledge and Systems Sciences, vol. 2, pp. 33-46, 2005.

[6] P. Jorion, Value at Risk: The New Benchmark for Managing Financial Market Risk, Two Penn Plaza, N.Y.: McGraw-Hill Trade, 2000.

[7] P. Giot and S. Laurent, "Market Risk in Commodity Markets: a VaR Approach," Energy Economics, vol. 25, pp. 435-457, 2003.

[8] J.C. Hotz., "An Evaluation of Value at Risk in the Alberta Pork Production Industry," master's thesis, Dept. of Rural Economics, Univ. of Alberta, Edmonton, Alberta, 2004.

[9] M.R. Manfredo, "Volatility Forecasting and Value-at-Risk: An Application to Cattle Feeding," PhD dissertation, Dept. of Agricultural Economics, Univ. of Illinois at Urbana-Champaign, Urbana, I.L., 1999.

[10] M. Odening and J. Hinrichs, "Assessment of Market Risk in Hog Production using Value-at-Risk and Extreme Value Theory," working paper, Humboldt University Berlin, 2002.

[11] P. Peterson and R. Leuthold, "A Portfolio Approach to Optimal Hedging for a Commercial Cattle Feedlot," The J.of Futures Markets, vol. 7, pp. 443-457, 1987.

[12] S. Makridakis, S. Anderson, A. Carbone, R. Fildes, R. Hibdon, M. Lewandowski, R. Newton, J. Parzen, and E. Winkler, "The Accuracy of Extrapolation (Time Series) Methods: Results of A Forecasting Competition," J. Forecasting, vol. 1, pp. 111-153, 1982.

[13] S. Makridakis and L. Robert, "Averages of Forecasts: Some Empirical Results,” Management Science, vol. 29, pp. 987-996, 1983.

[14] S. Makridakis, "Why Combining Works?" Int'l J. Forecasting, vol. 5, pp. 601-603, 1989 .
[15] P. Newbold and C.W. Granger, "Experience with Forecasting Univariate Time Series and the Combination of Forecasts (with Discussion)," J. the Royal Statistical Society: Series A (General), vol. 137, pp. 131-164, 1974.

[16] F.C. Palm and A. Zellner, "To Combine or Not to Combine? Issues of Combining Forecasts," J. Forecasting, vol. 11, pp. 687-701, 1992.

[17] K.J. He, C. Xie, S. Chen, and K.K. Lai, "Market Risk for Non Ferrous Metals: A Wavelet Based VaR Approach," First Int'l Conf. Knowledge Science, Engineering and Management, submitted for publication.

[18] D. Li, "Empirical Study of Investment Behaviour in Equity Markets using Wavelet Methods," PhD dissertation, Rensselaer Polytechnic Institute, Troy, N.Y., 2003.

[19] J.D. Cabedo and I. Moya, "Estimating Oil Price 'Value at Risk' Using Historical Simulation Approach," Energy Economics, vol. 25, pp. 239-253, 2003.

[20] T.H. Lee and B. Saltolu, "Assessing the Risk Forecasts for Japanese Stock Market," Japan and the World Economy, vol. 14, pp. 63-85, 2002.

[21] M. Pritsker, "The Hidden Dangers of Historical Simulation," $J$. Banking \& Finance, vol. 30, no. 2, pp. 561-582, 2006.

[22] M. Sadeghi and S. Shavvalpour, "Energy Risk Management and Value at Risk Modeling," Energy Policy, doi:10.1016/j.enpol.2005.07.004, March, 2006.

[23] R. Gençay, F. Selçuk, and A. Ulugülya, "High Volatility, Thick Tails and Extreme Value Theory in Value-at-Risk Estimation," Insurance: Math. and Economics, vol. 33, pp. 337-356, 2002.

[24] R. Gençay, F. Selçuk, and A. Ulugülya, "High Volatility, Thick Tails and Extreme Value Theory in Value-at-Risk Estimation," Insurance: Math. and Economics, vol. 33, pp. 337-356, 2002.

[25] P. Sadorsky, "Time-varying Risk Premiums in Petroleum Futures Prices," Energy Economics, vol. 24, pp. 539-556, 2002.

[26] J. Leskow, "The Impact of Stationarity Assessment on Studies of Volatility and Value-at-Risk," Math. and Computer Modeling, vol. 34, pp. 1213-1222, 2001.

[27] H. Akaike, "A New Look at the Statistical Model Identification," IEEE Trans. Automatic Control, vol. 19, pp. 716-723, 1974.

[28] R. Tsay, Analysis of Financial Time Series. West Sussex, England: John Wiley \& Sons, 2002.

[29] C. Brooks, Introductory econometrics for finance. Trumpinton Street, Cambridge: Cambridge University Press, 2002.

[30] J.a. Lopez, "Methods for Evaluating Value-at-Risk Estimates," Federal Reserve Bank of San Francisco Economic Review, vol. 2, pp. 3-15, 1999.

[31] A. Boggess and F.J. Narcowich, A First Course in Wavelets with Fourier Analysis, Upper Saddle River, N.J.: Prentice-Hall, 2001.

[32] S. Mallat, A Wavelet Tour of Signal Processing (2nd Edition), San Diego, CA: Elsevier Pte Ltd, 2003.

[33] D.B. Percival, A.T. Walden, Wavelet Methods for Time Series Analysis, Trumpinton Street, Cambridge: Cambridge Univ. Press, 2000.

[34] Y. Nievergelt, Wavelets Made Easy. 175 Fifth Avenue, N.Y.: Springer-Verlag, 1999.

[35] B.B. Hubbard, The World According to Wavelets: the Story of A Mathematical Technique in the Making (2nd Edition). Natrick, M.A.: A K Peters, 1998.

[36] A. Jensen and L.C. Anders, Ripples in Mathematics: the Discrete Wavelet Transform. Heidelberg, Berlin: Springer-Verlag, 2001.

[37] C. Cattani, "Haar Wavelet-based Technique for Sharp Jumps Classification," Mathematical and Computing Modeling, vol. 39, pp. 255-278, 2004.

[38] S. Kim and F. In, "The Relationship Between Stock Returns and Inflation: New Evidence From Wavelet Analysis," J. Empirical Finance, vol. 12, no. 3, pp. 435-444, 2005.

[39] J.B. Ramsey, "Wavelets in Economics and Finance: Past and Future," Studies in Non-linear Dynamics \& Econometrics, vol. 6, pp. 1-27, 2002.

[40] Z.R. Struzik, "Wavelet Methods in (Financial) Time-Series Processing," Physica A, vol. 296, pp. 307-319, 2001.

[41] M. Stevenson, "Filtering and Forecasting Spot Electricity Prices in the Increasingly Deregulated Australian Electricity Market," Research Paper Series 63, Quantitative Finance Research Centre, Univ. of Technology, Sydney, N.S.W., 2001. 
[42] Q.E. Booker, "Forecasting Swiss Franc/Dollar Exchange Rates: Comparing Collapsible Neural Networks to Recent Non Parametric Methods and Traditional Statistical Methods," PhD dissertation, Business School, the Univ. of Mississippi, University, M.S.: 1998.

[43] A. Bernabe, E. Martina, J. Alvarez-Ramirez, and C, Ibarra-Valdez, "A Multi-model Approach for Describing Crude Oil Price Dynamics," Physica A, vol. 338, pp. 567-584, 2004.

[44] S. Soltani, "On the Use of the Wavelet Decomposition for Time Series Prediction," Neurocomputing, vol. 48, pp. 267-277, 2002.

[45] F. Murtagh, J.L. Starck, and O. Renaud, "On Neuro-Wavelet Modeling," Decision Support Systems, vol. 37, pp. 475-484, 2004.

[46] A.J. Conejo, J. Contreras, R. Espinola, and M.A. Plazas, "Forecasting Electricity Prices for A Day-ahead Pool-based Electric Energy Market," Int'l J. Forecasting, vol. 21, pp. 435-462, 2004.

[47] C.I. Kim, I.K. Yu, and Y.H. Song, "Prediction of System Marginal Price of Electricity Using Wavelet Transform Analysis," Energy conversion and Management, vol. 43, pp. 1839-1851, 2002.

[48] H. Xu and T. Niimura, "Short-term Electricity Price Modeling and Forecasting Using Wavelets and Multivariate Time Series," Proc. 2004 Power Systems Conf. \& Exposition, pp. 1-5, 2004.

[49] S. Yousefi, H. Weinreich, and D. Reinarz, "Wavelet-based Prediction of Oil Prices," Chaos, Solutions and Fractals, vol. 25, pp. 265-275, 2005.

[50] V. Fernandez, "The CAPM and Value at Risk at Different Time-scales," Int'l Rev. of Financial Analysis, doi:10.1016/j.irfa.2005.02.004, March, 2006.

[51] V. Fernandez, "The International CAPM and a Wavelet-based Decomposition of Value at Risk," Studies of Nonlinear Dynamics \& Econometrics, vol. 9, no. 4, article 4, 2005.

[52] Y. Hamburger, "Wavelet-based Value at Risk Estimation: A Multiresolution Approach," master's thesis, Dept. of Informatics and Economics, Erasmus University, Erasmus, Rotterdam, 2003.

[53] A.S. Andreou, E.F. Georgopoulos, and S.D Likothanassis, "Exchange-Rates Forecasting: A Hybrid Algorithm Based on Genetically Optimized Adaptive Neural Networks," Computational Economics, vol. 20, pp. 191-210, 2002.

[54] L.A. Y, S.Y. Wong, and K.K. Lai, "A Novel Nonlinear Ensemble Forecasting Model Incorporating GLAR and ANN for Foreign Exchange Rates," Computers \& Operations Research, vol. 32, pp. 2523-2541, 2005.

[55] A. Nag, and A. Mitra, "Forecasting Daily Foreign Exchange Rates Using Genetically Optimized Neural Networks," J. Forecasting, vol. 21, pp. 501-511, 2002.

[56] W.A. Brock, D. Dechert, H. Scheinkman, and B. LeBaron, Nonlinear Dynamics, Chaos, and Instability: Statistical Theory and Economic Evidence. Cambridge, Mass: MIT Press, 1991. 